TAIWANESE JOURNAL OF MATHEMATICS

Vol. 2, No. 1, pp. 69-85, March 1998

\title{
NOTES ON MULTIVARIATE PRE-WAVELET DECOMPOSITION
}

\author{
Kuei-Fang Chang
}

\begin{abstract}
We introduce the Wiener space and then consider wavelets which are not necessarily compactly supported but have a decay condition at infinity. Under the Wiener condition, a wavelet and its bi-orthogonal wavelet have the same rate of decay at infinity and the same moment. Under a certain sufficient condition, we prove the existence of a pre-wavelet basis in the multi-dimensional case. Furthermore, pre-wavelets and their bi-orthogonal pre-wavelets have the same rate of decay at infinity and the same number of vanishing moments.
\end{abstract}

\section{INTRODUCTION}

We assume that $\phi$ belongs to the Wiener space $\mathcal{M}\left(\mathbb{R}^{n}\right)$ (see later) and has stable integer translates. If $\phi$ satisfies a 2-scale dilation equation with mask $a$, then $\phi$ admits a multi-resolution of $L^{2}\left(\mathbb{R}^{n}\right)$. In the 1-dimensional case, it is well-known that we can construct many pre-wavelets from $\phi$ (see [9]). But we want to construct a pre-wavelet in $\mathcal{M}(\mathbb{R})$ that allows us to extend our method to multivariate wavelet decomposition. In the multidimensional case, we have to find more than one function to construct a pre-wavelet basis. Jia and Micchelli [9] showed that the existence of a pre-wavelet basis is equivalent to the extensibility of a $2^{n}$-tuple $\left(\widetilde{a^{\nu}}\right)_{\nu \in I}$ over the commutative Banach algebra $\mathcal{A}$ containing all discrete Fourier transforms of $\ell^{1}$-sequences, where $I$ is the set of all extreme points of the unit cube $[0,1]^{n}$ and

$$
\widetilde{a^{\nu}}(\xi):=\sum_{\beta \in \mathbb{Z}^{n}} a_{2 \beta+\nu} e^{2 \pi i \beta \cdot \xi}, \quad \nu \in I ; \xi \in \mathbb{R}^{n} .
$$

Received July 26, 1995; revised June 17, 1997.

Communicated by P. Y. Wu.

1991 Mathematics Subject Classification: 42C15.

Key words and phrases: Wiener class, Poisson summation formula, pre-wavelet basis, Riesz basis, Box splines. 
For $\xi \in \mathbb{R}^{n}$, let $F(\xi)$ be the vector in $\mathbb{C}^{2^{n}}$ whose components are

$$
F(\xi):=\left(\widetilde{a^{\nu}}(\xi): \nu \in I\right)
$$

and let $F$ be Hölder continuous. Then it will be shown that $F$ is extensible, and hence a pre-wavelet basis can be constructed. In constructing a pre-wavelet basis, there is a difference between univariate and multivariate spaces; that is, if $n>1$ and $\phi$ in $\mathcal{M}\left(\mathbb{R}^{n}\right)$ admits a multi-resolution analysis in $L^{2}\left(\mathbb{R}^{n}\right)$, then there may not exist a pre-wavelet basis. But in the univariate case, this is always true. Under the Wiener condition, a wavelet and its bi-orthogonal wavelet have the same rate of decay at infinity and the same moment. Under a certain sufficient condition, we prove the existence of a pre-wavelet basis in the multi-dimensional case. Furthermore, pre-wavelets and their bi-orthogonal pre-wavelets have the same rate of decay at infinity and the same number of vanishing moments. An example of a non-tensor-product spline-wavelet (the bivariate box spline) will be given.

\section{Multivariate Pre-Wavelet Decomposition}

The Wiener class, denoted by $\mathcal{M}\left(\mathbb{R}^{n}\right)$, is defined as the set of all continuous functions on $\mathbb{R}^{n}$ satisfying the Wiener condition $\|f\|:=\|f\|_{w}+\|\hat{f}\|_{w}<\infty$, where

$$
\|f\|_{w}:=\sum_{k \in \mathbb{Z}^{n}} \max _{x \in[0,1]^{n}}|f(x+k)|<\infty .
$$

Poisson summation formula is a beautiful result incorporating ideas from both Fourier series and Fourier transforms, and it has had many applications to number theory, partial differential equations and probability theory as well as wavelets. The following theorem proves that every member of the Wiener class enjoys Poisson summation formula (see [7, p. 246]).

Theorem 2.1. Suppose that $f$ belongs to the Wiener class $\mathcal{M}\left(\mathbb{R}^{n}\right)$. Then the equalities

$$
\sum_{k \in \mathbb{Z}^{n}} f(x+k)=\sum_{k \in \mathbb{Z}^{n}} \hat{f}(k) e^{2 \pi i k x}
$$

and

$$
\sum_{k \in \mathbb{Z}^{n}} f(k) e^{2 \pi i k \xi}=\sum_{k \in \mathbb{Z}^{n}} \hat{f}(\xi+k)
$$

hold pointwise, and all four series converge absolutely and uniformly on $[0,1]^{n}$.

For convenience in notation, we define the following: 
Definition 2.2. Let $f$ be a complex-valued function on $\mathbb{R}^{n}$. We define the following operators $T_{a}, D_{a}$, and conjugation as follows:

Translation: $\left(T_{a} f\right)(x)=f(x+a)$ for $a \in \mathbb{R}^{n}$;

Dilation: $\left(D_{a} f\right)(x)=f(a x)$ for $a \in \mathbb{R}$;

Conjugation: $f^{*}(x)=\overline{f(-x)}$.

The following terminology "pre-wavelet" was first used by Battle [1].

Definition 2.3. A pre-wavelet is a square-integrable function $\psi$ such that $\psi\left(2^{j} \cdot+k\right)$ is orthogonal to $\psi\left(2^{m} \cdot+n\right)$ for all $k, n \in \mathbb{Z}$ and all integers $j \neq m$ and $\left\{\psi\left(2^{j} \cdot+k\right): j \in \mathbb{Z}, k \in \mathbb{Z}^{n}\right\}$ forms a Riesz basis.

Suppose that $\phi$ belongs to $\mathcal{M}\left(\mathbb{R}^{n}\right)$ and satisfies a 2-scale dilation equation

$$
\phi(x)=\sum_{k \in \mathbb{Z}^{n}} a_{k} \phi(2 x+k), \quad x \in \mathbb{R}^{n} .
$$

This implies that the sequence $a$ belongs to $\ell^{1}\left(\mathbb{Z}^{n}\right)$. We call the coefficient sequence $a$ of Equation (2.4) a mask. Let $\mathcal{V}_{0}:=\mathcal{V}^{\phi}, \mathcal{V}_{j}:=\left\{f\left(2^{j} \cdot\right): f \in \mathcal{V}_{0}\right\}$ and let $\mathcal{W}_{j}$ be the orthogonal complement of $\mathcal{V}_{j}$ in $V_{j+1}$. When $n>1$, we have to find more than one function to construct a pre-wavelet basis. Thus it is natural to consider the stability of the integer translates of functions. Let $\psi_{1}, \ldots, \psi_{s}$ be functions in $\mathcal{M}\left(\mathbb{R}^{n}\right)$. These functions give a linear operator

$$
L_{\psi_{1}, \ldots, \psi_{s}}:\left(a^{1}, \ldots, a^{s}\right) \mapsto \sum_{j=1}^{s} \psi_{j} *^{\prime} a^{j}, \quad a^{1}, \ldots, a^{s} \in \ell^{2}\left(\mathbb{Z}^{n}\right)
$$

Thus $L_{\psi_{1}, \ldots, \psi_{s}}$ is a bounded linear operator from $\left(\ell^{2}\left(\mathbb{Z}^{n}\right)\right)^{s}$ into $L^{2}\left(\mathbb{R}^{n}\right)$. The family of integer translates of $\psi_{j}$; that is, the family

$$
\left\{T_{k} \psi_{j}: k \in \mathbb{Z}^{n} ; j=1, \ldots, s\right\}
$$

is a stable family if there is a positive constant $A$ such that

$$
\left\|L_{\psi_{1}, \ldots, \psi_{s}}\left(a^{1}, \ldots, a^{s}\right)\right\|_{2} \geq A \sum_{j=1}^{s}\left\|a^{j}\right\|_{2}
$$

Jia and Micchelli [9] showed that the existence of a pre-wavelet basis for $\mathcal{W}_{0}$ is equivalent to the extensibility of the $2^{n}$-tuple $\left(\widetilde{a^{\nu}}\right)_{\nu \in I}$ over $\mathcal{A}$, where $I$ is the set of all extreme points of the unit cube $[0,1]^{n}$ and

$$
\widetilde{a^{\nu}}(\xi):=\sum_{\beta \in \mathbb{Z}^{n}} a_{2 \beta+\nu} e^{2 \pi i \beta \cdot \xi}, \quad \nu \in I ; \quad \xi \in \mathbb{R}^{n} .
$$


We denote by $\mathcal{A}$ the commutative Banach algebra consisting of all continuous periodic functions of period 1 having absolutely convergent Fourier series $[13$, p. 266].

Definition 2.6. An s-tuple of elements of $\mathcal{A},\left(p_{1}, \ldots, p_{s}\right)$ is extensible over $\mathcal{A}$ if there exist $s^{2}$ elements $p_{j k} \in \mathcal{A}(j, k=1,2, \ldots, s)$ such that $p_{1 k}=p_{k}$ for all $k$ and the matrix $\left(p_{j k}(z)\right)$ is nonsingular for all $z$ on the torus $T^{n}$.

Jia and Micchelli [10] gave a necessary condition for the extensibility of $\left(\tilde{b}_{\nu}\right)_{\nu \in I}$. Let $\Omega$ be a subset of $\mathbb{R}^{n}$. We say that $F: \Omega \rightarrow \mathbb{C}^{s}$ is Hölder continuous provided there is some $\rho \in(0,1]$ and constant $\lambda>0$ such that

$$
\|F(x)-F(y)\|_{\mathbb{C}^{s}} \leq \lambda\|x-y\|_{\mathbb{R}^{n}}^{\rho}, \quad x, y \in \Omega .
$$

Theorem $2.7[10]$. Let $F(\xi):=\left(f_{1}(\xi), \ldots, f_{s}(\xi)\right), \xi \in \mathbb{R}^{n}$ be a vector whose coordinates are trigonometric series

$$
f_{j}(\xi)=\sum_{k \in \mathbb{Z}^{n}} a_{k}^{j} e^{2 \pi i k \cdot \xi}, \quad \xi \in \mathbb{R}^{n}
$$

such that $F(\xi) \in \mathbb{C}^{s} \backslash\{0\}$ for all $\xi \in \mathbb{R}^{n}$. If $n<2 s-1$ and for some $\rho>0$,

$$
\sum_{k \in \mathbb{Z}^{n}}\|k\|^{\rho}\left|a_{k}^{j}\right|<\infty, \quad j=1, \ldots, s,
$$

then there is an invertible $s \times s$ matrix $M(\xi)$ for all $\xi \in \mathbb{R}^{n}$ such that the first row of $M(\xi)$ is $F(\xi)$ and the remaining rows are trigonometric polynomials.

Notice that Condition (2.8) ensures that the map $F$ is Hölder continuous. The proof of Theorem 2.7 is based on the idea that if $F$ is a Hölder mapping from some subset $\Omega$ of $\mathbb{R}^{n}$ into $\mathbb{R}^{s}$ and $n<s$, then $F(\Omega)$ has Lebesgue measure zero in $\mathbb{R}^{s}$. Indeed, Theorem 2.7 is a general version of Sard's Theorem (see [6, p. 204-205]). But the above theorem is not true for all continuous maps. It is an application of the Hahn-Mazuriewicz theorem (see [8, p. 129]) that there exists a continuous map of the unit interval $[0,1]$ onto the $n$-cube $[0,1]^{n}$ for all $n$.

We are interested in finding a sufficient condition such that the condition (2.8) holds. This is related to a Wiener-Tauberian Theorem with a "weight". Let $\sigma$ be a positive sequence satisfying the condition

$$
\sigma(k+l) \leq \sigma(k) \sigma(l) \quad \text { for all } k, l \in \mathbb{Z}^{n} .
$$

Following Gelfand, et al. [4] for one-dimension or Mitjagin [12] and Lei [11] for multi-dimensions, we denote by $W[\sigma]$ the set of all formal power series 
$f=\sum_{k \in \mathbb{Z}^{n}} a_{k} X^{k}$ for which

$$
\|f\|:=\sum_{k \in \mathbb{Z}^{n}}\left|a_{k}\right| \sigma(k)<\infty .
$$

Here $X$ is an indeterminate vector, $X:=\left(X_{1}, \ldots, X_{n}\right)$. It plays the role of a dummy variable in defining the formal power series. From (2.9) it follows that if $W[\sigma]$ contains two series $f=\sum_{k} a_{k} X^{k}$ and $g=\sum_{k} b_{k} X^{k}$, it also contains their formal product

$$
\sum_{m} c_{m} X^{m}=\sum_{m}\left(\sum_{l} a_{m-l} b_{l}\right) X^{m},
$$

and that

$$
\begin{aligned}
\|f g\| & =\sum_{m}\left|\sum_{l} a_{m-l} b_{l}\right| \sigma(m) \\
& \leq \sum_{m} \sum_{l}\left|a_{m-l}\right| \sigma(m-l)\left|b_{l}\right| \sigma(l) \\
& =\|f\|\|g\| .
\end{aligned}
$$

Thus $W[\sigma]$ is a Banach algebra having a unit under the formal operations on power series. Let $M$ be a maximal ideal of $W[\sigma]$. Then the residue class algebra $W[\sigma] / M$ is isomorphic to the complex number field. If we denote by $f(M)$ the complex number corresponding to $f \in W[\sigma]$ under this canonical mapping, then $|f(M)| \leq\|f\|$. For each $p \in\{1, \ldots, n\}$, the complex number $X_{p}(M)$ is non-zero since $X_{p}$ is invertible. Let $z$ be the element in $\mathbb{C}^{n}$ whose $p$-th component is $X_{p}(M)$. Then for each $k \in \mathbb{Z}^{n}, z^{k}=X^{k}(M)$ and

$$
\left|z^{k}\right|=\left|X^{k}(M)\right| \leq\left\|X^{k}\right\|=\sigma(k) .
$$

This leads us to consider the set

$$
S_{\sigma}:=\left\{z \in \mathbb{C}^{n} \backslash\{0\}:\left|z^{k}\right| \leq \sigma(k) \text { for all } k \in \mathbb{Z}^{n}\right\} .
$$

It is clear that for any formal power series $\sum_{k} a_{k} X^{k}$ in $W[\sigma]$, the power series $\sum_{k} a_{k} z^{k}$ converges uniformly in $z$ on $S_{\sigma}$. This implies that every element $f$ in $W[\sigma]$ defines a continuous function on $S_{\sigma}$, still denoted by $f$.

In our application, $\sigma$ is chosen by

$$
\sigma(x)=(1+\|x\|)^{\rho}, \quad x \in \mathbb{R}^{n},
$$

where $\rho$ is a positive number. Thus, $\sigma(x+y) \leq \sigma(x) \sigma(y)$. Lei [11] proved that $S_{\sigma}$ is exactly $T^{n}$ where $T^{n}$ is the torus in $\mathbb{C}^{n}$. The following theorem follows immediately from $[4$, Sect. 6 , Theorem 1$]$. 
Theorem 2.11. Let $\sigma$ be as in (2.10). If $f$ is a member of $W[\sigma]$ and if $G$ is analytic on a neighborhood of the range of the function on $T^{n}$, then there exists a member $g$ in $W[\sigma]$ such that $G(f(z))=g(z)$ for all $z \in T^{n}$.

This theorem is useful in estimating the decay rate of $\tilde{\phi}$ in terms of that of $\phi$, where $\phi$ is a stable function, and $\tilde{\phi}$ is a bi-orthogonal stable function with respect to $\phi$. We can show that $\tilde{\phi}$ has the same decay rate.

Theorem 2.12. Let $\rho$ be a positive number. If $\phi$ belongs to $C\left(\mathbb{R}^{n}\right)$ with $\|\sigma \phi\|_{w}<\infty$ and has stable shifts, then there exists a sequence $b$ for which the formal power series $g:=\sum_{k} b_{k} X_{k}$ is in $W[\sigma]$ and $\tilde{\phi}:=\phi *^{\prime} b$ has stable shifts with $\|\sigma \tilde{\phi}\|_{w}<\infty$.

Proof. Let $a$ be the sequence given by $a_{k}:=\phi * \phi^{*}(k)$ and let $f$ be the formal power series $\sum_{k} a_{k} X^{k}$. We want to show that $f$ belongs to $W[\sigma]$. For any $k \in \mathbb{Z}^{n}$ and $x \in \mathbb{R}^{n}$, we have

$$
\begin{aligned}
\sum_{k} \sigma(k)\left|a_{k}\right| & \leq \sum_{k} \sigma(k) \int_{\mathbb{R}^{n}}|\phi(x) \phi(x+k)| d x \\
& \leq \sum_{k} \int_{\mathbb{R}^{n}}|(\sigma \phi)(x)||(\sigma \phi)(x+k)| d x \\
& \leq \sum_{k} \sum_{l} \int_{[0,1]^{n}}|(\sigma \phi)(x+l)||(\sigma \phi)(x+k+l)| d x \\
& \leq\|\sigma \phi\|_{w}^{2}<\infty .
\end{aligned}
$$

Thus, $f \in W[\sigma]$. Since $\phi$ has stable shifts, $f(z)$ is positive on $T^{n}$ (see $[9$, Theorem 3.3]). By Theorem 2.11, there exists a sequence $b$ for which the formal power series $g:=\sum_{k} b_{k} X^{k}$ is in $W[\sigma]$ and

$$
\frac{1}{f(z)}=g(z) \text {. }
$$

This implies that $\tilde{\phi}=\phi *^{\prime} b$ is bi-orthogonal with respect to $\phi$. Finally, in order to show $\|\sigma \tilde{\phi}\|_{w}<\infty$, we note that

$$
\begin{aligned}
\sum_{k} \max _{x \in[0,1]^{n}}|(\sigma \tilde{\phi})(x+k)| & \leq \sum_{k} \sum_{l} \max _{x \in[0,1]^{n}}|(\sigma \phi)(x+k+l)|\left|\sigma(l) b_{l}\right| \\
& \leq\|\sigma \phi\|_{w}\|g\|<\infty .
\end{aligned}
$$

That is $\|\sigma \tilde{\phi}\|_{w}<\infty$.

Corollary 2.13. Assume the hypotheses of Theorem 2.12 and suppose $\phi$ satisfies a 2-scale dilation equation with mask a; that is $\phi(x / 2)=\sum_{k} a_{k} \phi(x+$ $k$ ). Then the condition (2.8) holds. 
Proof. Define a function $h$ by

$$
h:=\frac{\sigma}{D_{1 / 2} \sigma},
$$

where $D_{1 / 2}$ is the dilation operation by $1 / 2$. Thus $h$ is bounded and continuous on $\mathbb{R}^{n}$. By Theorem 2.12, it follows that $\tilde{\phi}$ satisfies $\|\sigma \tilde{\phi}\|_{w}<\infty$ and then

$$
\begin{aligned}
\sum_{k} \sigma(k)\left|a_{k}\right| & \leq \sum_{k} \sigma(k) \int_{\mathbb{R}^{n}}\left|\phi\left(\frac{x}{2}\right) \tilde{\phi}(x+k)\right| d x \\
& \leq \sum_{k} \int_{\mathbb{R}^{n}}|\sigma(x) \phi(x+k)||(\sigma \tilde{\phi})(x+k)| d x \\
& \leq\|h\|_{\infty}\|\sigma \tilde{\phi}\|_{w} \int_{[0,1]^{n}} \sum_{k}\left|(\sigma \tilde{\phi})\left(\frac{x}{2}+l\right)\right| d x \\
& \leq\|h\|_{\infty}\|\sigma \tilde{\phi}\|_{w}^{2}<\infty .
\end{aligned}
$$

Consequently, the condition (2.8) holds.

Now we employ Theorem 2.7 to construct pre-wavelet functions in $\mathcal{M}\left(\mathbb{R}^{n}\right)$. The following lemmas and theorems are taken from [2, Sect. 4.3].

Lemma 2.14. Suppose that $F$ is defined by

$$
F(\xi):=\left(\widetilde{a^{\nu}}(\xi): \nu \in I\right), \quad \xi \in \mathbb{R}^{n},
$$

where $\widetilde{a^{\nu}}$ are in $(2.5)$. Then $F$ is a continuous map from $[0,1]^{n}$ into $\mathbb{C}^{s} \backslash\{0\}$ with $s=2^{n}$.

Lemma 2.15. Assume the hypotheses of Corollary 2.13 and Lemma 2.14. Then there exist $2^{n}$ functions $\phi_{\nu}, \nu \in I$, in $\mathcal{M}\left(\mathbb{R}^{n}\right)$ such that

(i) $\phi_{\nu} \in \mathcal{V}^{\phi}, \phi_{0}=\phi$ and $\mathcal{V}_{1}=\sum_{\nu \in I} \mathcal{V}^{\phi_{\nu}}$,

(ii) the integer translates of $\phi_{\nu}, \nu \in I$, form a stable family.

The following lemma is proved by a Gram-Schmidt orthogonalization process (see [9]). Define

$$
\mathcal{V}^{\left\{\phi_{1}, \ldots, \phi_{s}\right\}}:=\left\{\sum_{j=1}^{s} d^{j} *^{\prime} \phi_{j}: d^{j} \in \ell^{2}\left(\mathbb{Z}^{n}\right), j=1, \ldots, s\right\} .
$$

Lemma 2.16. Suppose that $\phi_{1}, \ldots, \phi_{s} \in \mathcal{M}\left(\mathbb{R}^{n}\right)$ have stable integer translates. Then there are $\psi_{1}, \ldots, \psi_{s} \in \mathcal{M}\left(\mathbb{R}^{n}\right) \cap \mathcal{V}^{\left\{\phi_{1}, \ldots, \psi_{s}\right\}}$ and an $s \times s$ lowertriangular matrix $N=\left(d_{i j}\right)$, where $d_{i j} \in \ell^{1}\left(\mathbb{Z}^{n}\right)$ such that $\psi_{i}=\sum_{j=1}^{s} d_{i j} *^{\prime} \phi_{j}$, and $d_{i i}>0$. Moreover 
(i) $\psi_{1}=\phi_{1},\left(\tilde{d}_{11}=1\right)$,

(ii) $\mathcal{V}^{\left\{\phi_{1}, \ldots, \phi_{s}\right\}}=\mathcal{V}^{\left\{\psi_{1}, \ldots, \psi_{s}\right\}}$,

(iii) $\mathcal{V}^{\psi_{j}}(j=1, \ldots, s)$ are mutually orthogonal subspaces of $L^{2}\left(\mathbb{R}^{n}\right)$.

Define

$$
\mathcal{V}_{j}^{\psi}:=\left\{\sum_{k \in \mathbb{Z}^{n}} b_{k} D_{2 j} T_{k} \psi: b \in \ell^{2}\left(\mathbb{Z}^{n}\right)\right\} .
$$

Combining Lemmas 2.14-16, we have the following theorem.

Theorem 2.17. Suppose that $\phi$ belongs to $\mathcal{M}\left(\mathbb{R}^{n}\right)$ with $\|\sigma \phi\|_{w}<\infty$ (as in Theorem 2.9) and has stable shifts. Let $\phi$ satisfy the 2-scale dilation equation (2.1). Then there are $2^{n}$ functions $\psi_{\nu}, \nu \in I$, in $\mathcal{M}\left(\mathbb{R}^{n}\right)$ such that

(i) $\psi_{\nu} \in \mathcal{V}_{1}, \psi_{0}=\phi$,

(ii) $\mathcal{V}_{j}^{\psi_{\nu}} \perp \mathcal{V}_{j}^{\psi_{\nu^{\prime}}}, \nu \neq \nu^{\prime} \in I ; j \in \mathbb{Z}$,

(iii) $\mathcal{W}_{j}=\oplus_{\nu \in I \backslash\{0\}} \mathcal{V}_{j}^{\psi_{\nu}}, j \in \mathbb{Z}$

(iv) $L^{2}\left(\mathbb{R}^{n}\right)=\oplus_{j \in \mathbb{Z}} \mathcal{W}_{j}$.

It follows from Theorem 2.7 that $F$ is extensible since $n<2^{n+1}-1=2 s-1$; that is, there is an invertible $s \times s$ matrix $M(\xi), \xi \in[0,1]^{n}$,

$$
M(\xi)=\left(\tilde{m}_{\nu \nu^{\prime}}\right)_{\nu, \nu^{\prime} \in I},
$$

where $\tilde{m}_{\nu \nu^{\prime}}$ is defined as in (2.5). Indeed, $m_{0 \nu}=a^{\nu}, \nu \in I$, and the remaining entries are trigonometric polynomials. Using this matrix we define functions

$$
\phi_{\nu}:=\sum_{\nu^{\prime} \in I} m_{\nu \nu^{\prime}} *^{\prime} D_{2} T_{\nu^{\prime}} \phi, \quad \nu \in I .
$$

Thus we obtain

$$
\phi_{0}=\sum_{\nu \in I} a^{\nu} *^{\prime} D_{2} T_{\nu} \phi=a *^{\prime} D_{2} \phi=\phi,
$$

and $\phi_{\nu}$ belongs to $\mathcal{M}\left(\mathbb{R}^{n}\right)$ for all $\nu \in I$.

We have a similar result in Corollary 2.13; that is, for any $f \in L^{2}\left(\mathbb{R}^{n}\right), f$ can be recovered by the pre-wavelet basis

$$
\left\{\psi_{j k}^{\nu}: j \in \mathbb{Z}, k \in \mathbb{Z}^{n}, \text { and } \nu \in I \backslash\{0\}\right\} .
$$

Corollary 2.19. Under the hypotheses of Theorem 2.17, there are prewavelet functions $\psi_{\nu}, \nu \in I \backslash\{0\}$, such that for any $f \in L^{2}\left(\mathbb{R}^{n}\right)$,

$$
f=\sum_{\nu \in I \backslash\{0\}} \sum_{j \in \mathbb{Z}} \sum_{k \in \mathbb{Z}^{n}}\left(f, \widetilde{\psi_{j k}^{\nu}}\right) \psi_{j k}^{\nu}=\sum_{\nu \in I \backslash\{0\}} \sum_{j \in \mathbb{Z}} \sum_{k \in \mathbb{Z}^{n}}\left(f, \psi_{j k}^{\nu}\right) \widetilde{\psi_{j k}^{\nu}}
$$


in the $L^{2}$-sense, where $\widetilde{\psi_{\nu}}$ is the dual of $\psi$.

It is natural to ask what conditions can guarantee that this family

$$
\left\{\psi_{j k}^{\nu}: j \in \mathbb{Z}, k \in \mathbb{Z}^{n}, \text { and } \nu \in I \backslash\{0\}\right\}
$$

is a Riesz basis for $L^{2}\left(\mathbb{R}^{n}\right)$; that is, there are two positive constants $A$ and $B$ such that the linear operator

$$
\left(a^{\nu}: \nu \in I \backslash\{0\}\right) \mapsto \sum_{\nu \in I \backslash\{0\}} \sum_{j \in \mathbb{Z}} \sum_{k \in \mathbb{Z}^{n}} a_{j k}^{\nu} \psi_{j k}^{\nu}
$$

is bounded by

$$
A \sum_{\nu \in I \backslash\{0\}}\left\|a^{\nu}\right\|_{2} \leq\left\|\sum_{\nu \in I \backslash\{0\}} \sum_{j \in \mathbb{Z}} \sum_{k \in \mathbb{Z}^{n}} a_{j k}^{\nu} \psi_{j k}^{\nu}\right\|_{2} \leq B \sum_{\nu \in I \backslash\{0\}}\left\|a^{\nu}\right\|_{2}
$$

for all $a^{\nu} \in \ell^{2}\left(\mathbb{Z} \times \mathbb{Z}^{n}\right)$. The following is an application to Theorem 2.17.

Theorem 2.20. Adopt the hypotheses of Theorem 2.18 and suppose that $\left\{\psi_{\nu}\right\}$ is a pre-wavelet basis $\psi_{\nu}(\nu \in I \backslash\{0\})$ having stable shifts. Then $\left\{\psi_{j k}^{\nu}\right\}_{\nu, j, k}$ is a Riesz basis (hence a frame) and $\left\{\tilde{\psi}_{j k}^{\nu}\right\}_{\nu, j, k}$ is the dual frame with $\left(\psi_{j k}^{\nu}, \tilde{\psi}_{j^{\prime} k^{\prime}}^{\nu^{\prime}}\right)$ $=\delta_{\nu \nu^{\prime}} \delta_{j j^{\prime}} \delta_{k k^{\prime}}$.

Next, we will prove that $\phi$ and $\psi_{\nu}$ have the same regularity.

Theorem 2.21. If $\phi$ belongs to $\mathcal{M}\left(\mathbb{R}^{n}\right)$ with $\|\sigma \phi\|_{w}+\|\sigma \hat{\phi}\|_{w}<\infty$ and has stable shifts, then $\left\|\sigma \psi_{\nu}\right\|_{w}<\infty$ and $\psi_{\nu} \in C^{\rho}\left(\mathbb{R}^{n}\right) \cap \mathcal{M}\left(\mathbb{R}^{n}\right)$ for all $\nu \in I \backslash\{0\}$.

Proof. Assume that $m_{\nu \nu^{\prime}}=\left(m_{\nu \nu^{\prime}}^{k}\right)_{k \in \mathbb{Z}^{n}}$. Then

$$
\begin{aligned}
\left\|\sigma \phi_{\nu}\right\|_{w} & =\sum_{k \in \mathbb{Z}^{n}} \max _{x \in[0,1]^{n}}\left|\sigma(x+k) \phi_{\nu}(x+k)\right| \\
& =\sum_{k \in \mathbb{Z}^{n}} \max _{x \in[0,1]^{n}}\left|\sigma(x+k) \sum_{\nu^{\prime} \in I} m_{\nu \nu^{\prime}} *^{\prime} \phi\left(2(x+k)+\nu^{\prime}\right)\right| \\
& =\sum_{k \in \mathbb{Z}^{n}} \max _{x \in[0,1]^{n}}\left|\sigma(x+k) \sum_{\nu^{\prime} \in I} \sum_{l \in \mathbb{Z}^{n}} m_{\nu \nu^{\prime}}^{l} \phi\left(2(x+k)+\nu^{\prime}+l\right)\right| \\
& \leq \sum_{l \in \mathbb{Z}^{n}} \sum_{\nu^{\prime} \in I}\left|m_{\nu \nu^{\prime}}^{l}\right| \sum_{k \in \mathbb{Z}^{n}} \max _{x \in[0,1]^{n}}\left|\sigma(x+k) \phi\left(2 x+2 k+\nu^{\prime}+l\right)\right| \\
& \leq\left(\sum_{\nu^{\prime} \in I} \sum_{l \in \mathbb{Z}^{n}}\left|m_{\nu \nu^{\prime}}^{l}\right|\right) \sum_{k, l \in \mathbb{Z}^{n}} \max _{x \in[0,1]^{n}}|\sigma(2 x+2 k) \phi(2 x+2 k+l)|<\infty
\end{aligned}
$$


since $m_{\nu, \nu^{\prime}} \in \mathcal{A}$. Finally, we want to show that $\left\|\sigma \hat{\psi}_{\nu}\right\|_{w}<\infty$. This is dependent on

$$
\begin{aligned}
\hat{\psi}_{\nu}(\xi) & =\int_{\mathbb{R}^{n}} \sum_{\nu^{\prime} \in I} m_{\nu \nu^{\prime}} *^{\prime} \phi\left(2 x+\nu^{\prime}\right) e^{-2 \pi i x \xi} d x \\
& =\int_{\mathbb{R}^{n}} \sum_{l \in \mathbb{Z}^{n}} \sum_{\nu^{\prime} \in I} m_{\nu, \nu^{\prime}}^{l} \phi\left(2 x+\nu^{\prime}+l\right) e^{-2 \pi i x \xi} d x \\
& =\sum_{l \in \mathbb{Z}^{n}} \sum_{\nu^{\prime} \in I} m_{\nu, \nu^{\prime}}^{l} \int_{\mathbb{R}^{n}} \phi\left(2 x+\nu^{\prime}+l\right) e^{-2 \pi i x \xi} d x \\
& =\sum_{l \in \mathbb{Z}^{n}} \sum_{\nu^{\prime} \in I} m_{\nu, \nu^{\prime}}^{l} \int_{\mathbb{R}^{n}} \phi(y) e^{-2 \pi i\left(\frac{y-\nu^{\prime}-l}{2}\right) \xi} \frac{1}{2^{n}} d y \\
& =\sum_{l \in \mathbb{Z}^{n}} \sum_{\nu^{\prime} \in I} m_{\nu, \nu^{\prime}}^{l} \frac{1}{2^{n}} \int_{\mathbb{R}^{n}} \phi(y) e^{-2 \pi y\left(\frac{\xi}{2}\right)} e^{\pi i\left(\nu^{\prime}+l\right) \xi} d y \\
& =H(\xi) \hat{\phi}\left(\frac{\xi}{2}\right)
\end{aligned}
$$

where $H(\xi):=\frac{1}{2^{n}} \sum_{l \in \mathbb{Z}^{n}} \sum_{\nu^{\prime} \in I} m_{\nu \nu^{\prime}}^{l} e^{\pi i\left(\nu^{\prime}+l\right) \xi}$. The function $H(\xi)$ is continuous on $[0,1]^{n}$. Thus, $C=\max _{\xi \in[0,1]^{n}}|H(\xi)|<\infty$ and

$$
\begin{aligned}
\left\|\sigma \hat{\psi}_{\nu}\right\|_{w} & =\sum_{k \in \mathbb{Z}^{n}} \max _{\xi \in[0,1]^{n}}\left|\sigma(\xi+k) \hat{\psi}_{\nu}(\xi+k)\right| \\
& =\sum_{k \in \mathbb{Z}^{n}} \max _{\xi \in[0,1]^{n}}\left|\sigma(\xi+k) H(\xi+k) \hat{\phi}\left(\frac{\xi+k}{2}\right)\right| \\
& \leq C \sum_{k \in \mathbb{Z}^{n}} \max _{\xi \in[0,1]^{n}}\left|\sigma(\xi+k) \hat{\phi}\left(\frac{\xi+k}{2}\right)\right| \\
& \leq C \sum_{k \in \mathbb{Z}^{n}} \max _{\xi \in[0,1]^{n}}|\sigma(\xi+k) \hat{\phi}(\xi+k)|<\infty .
\end{aligned}
$$

This implies that $\psi_{\nu} \in C^{\rho}\left(\mathbb{R}^{n}\right)$ and hence $\psi_{\nu} \in C^{\rho}\left(\mathbb{R}^{n}\right) \cap \mathcal{M}\left(\mathbb{R}^{n}\right)$.

It is well known that if $\phi$ has stable shifts, then so does its dual $\tilde{\phi}$. We will show that if $\left\{\psi_{\nu}\right\}_{\nu \in I}$ has stable shifts, then so does $\left\{\tilde{\psi}_{\nu}\right\}_{\nu \in I}$. In what follows it is more convenient to use vector notation. Therefore, let

$$
\psi=\left(\psi_{\nu}: \nu \in I \backslash\{0\}\right)^{T}
$$

and let

$$
\Theta(\omega):=\sum_{k \in \mathbb{Z}^{n}} \hat{\psi}(\omega+k) \hat{\psi}^{*}(\omega+k),
$$

where $\hat{\psi}(\omega):=\int_{\mathbb{R}^{n}} e^{-2 \pi i \omega x} \psi(x) d x$ denotes the Fourier transform of $\psi$. Then the matrix $\Theta(\omega)$ is nonnegative-definite for all $\omega$. Using Poisson summation 
formula

$$
\sum_{k}\left(\psi * \psi^{*}\right)(k) e^{2 \pi i \omega k}=\sum_{k} \hat{\psi}(\omega+k) \hat{\psi}^{*}(\omega+k),
$$

we have

$$
\begin{aligned}
\Theta(\omega) & =\sum_{k}\left(\psi * \psi^{*}\right)(k) e^{2 \pi i \omega k} \\
& =\sum_{k}\left(\int_{\mathbb{R}^{n}} \psi(y-k) \psi^{*}(y) d y\right) e^{2 \pi i \omega k} .
\end{aligned}
$$

We next extend the result of [5] about the necessary and sufficient condition for the set of integer translate of $\psi_{\nu}$ 's to be a Riesz basis of $V_{0}$ in the Wiener class. Since the proof is similar, we omit it.

Theorem 2.22. The collection $B_{\psi}=\left\{\psi_{\nu}(\cdot+k): \nu \in I \backslash\{0\} ; k \in \mathbb{Z}^{n}\right\}$ forms a Riesz basis for $W_{0}$ if and only if $\Theta(\omega)$ is positive-definite for all $\omega$.

Assume that $\left\{T_{n} \psi_{\nu}: \nu \in I \backslash\{0\} ; n \in \mathbb{Z}^{n}\right\}$ is a Riesz base of $W_{0}$. If we define $r:=2^{n}-1$ and

$$
\tilde{\psi}=\left(\tilde{\psi}_{1}, \ldots, \tilde{\psi}_{r}\right)^{T}
$$

by

$$
\hat{\tilde{\psi}}=\Theta^{-1} \hat{\psi}
$$

where $\Theta=\left(\Theta_{\nu \nu^{\prime}}\right)$ and $\Theta^{-1}=\left(\Theta_{\nu \nu^{\prime}}^{-1}\right)$. Then by Parseval's Identity, we have $\left\langle\psi_{\nu}, T_{n} \tilde{\psi}_{\nu^{\prime}}\right\rangle=\delta_{\nu, \nu^{\prime}} \delta_{n, 0}$ (see [5]). Thus, $\tilde{\psi}$ is a dual vector of $\psi$. Moreover, we can show that $\psi_{\nu}$, is in $\mathcal{M}\left(\mathbb{R}^{n}\right)$ for all $j=1,2, \ldots, r$. Since $\Theta(w)$ is positivedefinite and periodically continuous for all $w$, all the entries of $\Theta^{-1}(w)$ are in $\mathcal{M}\left(\mathbb{R}^{n}\right)$. By Poisson summation formula, we have

$$
\Theta^{-1}(w)=\sum_{n \in \mathbb{Z}^{n}} e_{n} e^{-2 \pi i n w}
$$

where $\left(e_{n}\right)_{n \in \mathbb{Z}^{n}}$ is a sequence of $r \times r$ matrices with entries in $l^{1}\left(\mathbb{Z}^{n}\right)$. From (2.23) and (2.24), we get

$$
\tilde{\psi}(x)=\sum_{l \in \mathbb{Z}^{n}} e_{l} \psi(x-l)
$$

This implies that $\tilde{\psi}_{\nu}$ is in $\mathcal{M}\left(\mathbb{R}^{n}\right)$ for all $\nu \in I \backslash\{0\}$.

Corollary 2.26. If $\left\{\psi_{\nu}, \nu \in I \backslash\{0\}\right\}$ is stable, then $\left\{\tilde{\psi}_{\nu}, \nu \in I \backslash\{0\}\right\}$ has stable shifts. 
Proof. By Theorem 2.22, it suffices to show that

$$
\tilde{\Theta}(w):=\sum_{k \in \mathbb{Z}^{n}} \hat{\tilde{\psi}}(w+h) \widehat{\tilde{\psi}^{*}}(w+k)
$$

is positive-definite for all $w$. We have

$$
\begin{aligned}
x^{*} \tilde{\Theta}(w) x & =\sum_{k \in \mathbb{Z}^{n}} x^{*} \hat{\tilde{\psi}}(w+k) \widehat{\tilde{\psi}^{*}}(w+k) x \\
& =\sum_{k \in \mathbb{Z}^{n}} x^{*} \Theta^{-1}(w) \hat{\psi}(w+k) \hat{\psi}^{*}(w+k) \Theta^{-1}(w) x=x^{*} \Theta^{-1}(w) x>0
\end{aligned}
$$

for all $0 \neq x \in \mathbb{C}^{r}$. Thus $\tilde{\Theta}(w)$ is positive-definite for all $w$.

Corollary 2.27. Let $\phi$ belong to $\mathcal{M}\left(\mathbb{R}^{n}\right)$ with $\|\sigma \hat{\phi}\|_{w}<\infty$ and let $\phi$ have stable shifts. Then $\tilde{\psi}_{\nu} \in C^{\rho}\left(\mathbb{C}^{n}\right) \cap \mathcal{M}\left(\mathbb{R}^{n}\right)$.

Proof. By Theorem 2.21, we have

$$
\left\|\sigma \hat{\tilde{\psi}}_{\nu}\right\|_{w}=\left\|\sum_{\nu^{\prime}=1}^{r} \sigma \Theta_{\nu \nu^{\prime}}^{-1} \hat{\psi}_{\nu^{\prime}}\right\|_{w} \leq M_{\nu} \sum_{\nu^{\prime}=1}^{r}\left\|\sigma \hat{\psi}_{\nu^{\prime}}\right\|_{w}<\infty
$$

where $M_{\nu}=\max _{\xi \in[0,1]^{n}}\left\{\Theta_{\nu \nu^{\prime}}^{-1}(\xi): \nu^{\prime} \in I \backslash\{0\}\right\}$ since $\Theta_{\nu \nu^{\prime}}^{-1} \in \mathcal{A}$. Thus, $\tilde{\psi}_{\nu} \in$ $C^{\rho}\left(\mathbb{R}^{n}\right) \cap \mathcal{M}\left(\mathbb{R}^{n}\right)$.

By (2.18), we have the equation

$$
\psi\left(\frac{x}{2}\right)=\sum_{n \in \mathbb{Z}^{n}} Q_{n} \psi(x+n),
$$

where $\left(Q_{n}\right)_{n \in \mathbb{Z}^{n}}$ is a sequence of $r \times r$ matrices with entries in $l^{2}\left(\mathbb{Z}^{n}\right)$. It follows from Theorem 2.7 that $\left(Q_{n}\right)_{n \in \mathbb{Z}^{n}}$ belongs to $l^{1}\left(\mathbb{Z}^{n}\right)$.

Theorem 2.29. Let $\psi_{\nu}$ belong to $\mathcal{M}\left(\mathbb{R}^{n}\right)$ and satisfy Equations (2.18). If $Q_{n}=\left(Q_{\nu \nu^{\prime}}(n)\right)$, then $\left\{Q_{\nu \nu^{\prime}}(n)\right\}_{n \in \mathbb{Z}^{n}}$ must be in $l^{1}\left(\mathbb{Z}^{n}\right)$ for all $\nu, \nu^{\prime} \in I \backslash\{0\}$.

Next, we will prove that $\tilde{\psi}_{\nu}$ has the same decay rate as $\psi_{\nu}$ for all $\nu \in I \backslash\{0\}$. Recall that $\|\sigma f\|_{w}=\sum_{k \in \mathbb{Z}^{n}} \max _{x \in[0,1]^{n}}|\sigma(x+k) f(x+k)|$.

Theorem 2.30. Suppose that $\psi_{\nu}$ belongs to $\mathcal{M}\left(\mathbb{R}^{n}\right)$ with $\left\|\sigma \psi_{\nu}\right\|_{w}<\infty$ for all $\nu \in I \backslash\{0\}$. Let $a_{\nu \nu^{\prime}}(k)=\psi_{\nu} * \psi_{\nu^{\prime}}^{*}(k)$. Then $\sum_{k \in \mathbb{Z}^{n}} \sigma(k)\left|a_{\nu \nu^{\prime}}(k)\right|<\infty$ for all $\nu, \nu^{\prime} \in I \backslash\{0\}$. 
Proof.

$$
\begin{aligned}
\sum_{k \in \mathbb{Z}^{n}} \sigma(k)\left|a_{\nu \nu^{\prime}}(k)\right| & =\sum_{k \in \mathbb{Z}^{n}} \sigma(k)\left|\int_{\mathbb{R}^{n}} \psi_{\nu}(x) \psi_{\nu^{\prime}}(x+k)\right| d x \\
& \leq \sum_{k \in \mathbb{Z}^{n}} \int_{\mathbb{R}^{n}}\left|\left(\sigma \psi_{\nu}\right)(x)\right|\left|\left(\sigma \psi_{\nu^{\prime}}\right)(x+k)\right| d x \\
& \leq \sum_{k \in \mathbb{Z}^{n}} \sum_{l \in \mathbb{Z}^{n}} \int_{[0,1]^{n}}\left|\left(\sigma \psi_{\nu}\right)(x+l)\right|\left|\left(\sigma \psi_{\nu}^{\prime}\right)(x+k+l)\right| d x \\
& \leq\left\|\sigma \psi_{\nu}\right\|_{w}\left\|\sigma \psi_{\nu}^{\prime}\right\|_{w}<\infty
\end{aligned}
$$

Using Theorem 2.30 we can prove that $\psi_{\nu}$ and $\tilde{\psi}_{\nu}$ have the same decay rate.

Theorem 2.31. If $\psi_{\nu}$ belongs to $\mathcal{M}\left(\mathbb{R}^{n}\right)$ with $\left\|\sigma \psi_{\nu}\right\|_{w}<\infty$, then the function $\tilde{\psi}_{\nu}$ defined by Equation (2.23) satisfies $\left\|\sigma \tilde{\psi}_{\nu}\right\|_{w}<\infty$ for all $\nu \in I \backslash\{0\}$.

Proof. By Poisson summation formula, we know that

$$
\Theta(w)=\sum_{k \in \mathbb{Z}^{n}} \psi * \psi^{*}(k) e^{2 \pi i w k} .
$$

Let $\Theta_{\nu \nu^{\prime}}(w)=\sum_{k \in \mathbb{Z}^{n}} a_{\nu \nu^{\prime}}(k) e^{2 \pi i w k}$, where $a_{\nu \nu^{\prime}}(k)=\psi_{\nu} * \psi_{\nu^{\prime}}^{*}(k)$. Using Theorem 2.30, we obtain a sequence $\left\{a_{\nu \nu^{\prime}}(k)\right\}_{k \in \mathbb{Z}^{n}}$ satisfying

$$
\sum_{k \in \mathbb{Z}^{n}} \sigma(k)\left|a_{\nu \nu^{\prime}}(k)\right|<\infty
$$

for all $\nu, \nu^{\prime} \in I \backslash\{0\}$. Since $\Theta(w)$ is positive-definite and periodically continuous for all $w$, we have $\operatorname{det}(\Theta(w)) \geq m$ for all $w$, where $m>0$. If we let $A_{\nu \nu^{\prime}}$ be the matrix obtained from $\Theta$ by deleting its $\nu$-th row and $\nu^{\prime}$-th column, then

$$
\Theta_{\nu \nu^{\prime}}^{-1}(w)=\frac{(-1)^{|\nu|+\left|\nu^{\prime}\right|} \operatorname{det}\left(A_{\nu^{\prime} \nu}(w)\right)}{\operatorname{det}(\Theta(w))} \leq \frac{1}{m}(-1)^{|\nu|+\left|\nu^{\prime}\right|} \operatorname{det}\left(A_{\nu^{\prime} \nu}(w)\right),
$$

where $|\nu|$ denotes the sum of its components. Thus $\Theta_{\nu \nu^{\prime}}^{-1}$ is bounded. Moreover, we have that $\Theta_{\nu \nu^{\prime}}^{-1}$ is a finite linear combination of the entries of $\Theta(w)$. Let $\Theta_{\nu \nu^{\prime}}^{-1}(w)=\sum_{k \in \mathbb{Z}^{n}} c_{\nu \nu^{\prime}}(k) e^{-2 \pi i w k}$. Then

$$
\sum_{k \in \mathbb{Z}^{n}} \sigma(k)\left|c_{\nu \nu^{\prime}}(k)\right|<\infty
$$

for all $\nu, \nu^{\prime} \in I \backslash\{0\}$ since $W(\sigma)$ is a Banach algebra. Using Equations (2.24) and (2.25), we have

$$
\tilde{\psi}(x)=\sum_{l \in \mathbb{Z}^{n}} e(l) \psi(x-l),
$$


where $e(l)=\left(c_{\nu \nu^{\prime}}(l)\right)_{\nu \nu^{\prime}=1}^{r}$. Hence for all $\nu=1,2, \ldots, r$, we obtain

$$
\begin{aligned}
\left\|\sigma \tilde{\psi}_{\nu}\right\|_{w} & =\sum_{m \in \mathbb{Z}^{n}} \max _{x \in[0,1]^{n}}\left|\sigma(x+m) \sum_{l \in \mathbb{Z}^{n}} \sum_{\nu^{\prime}=1}^{r} c_{\nu \nu^{\prime}}(l) \psi_{\nu^{\prime}}(x-l+m)\right| \\
& \leq \sum_{m \in \mathbb{Z}^{n}} \sum_{l \in \mathbb{Z}^{n}} \sum_{\nu^{\prime}=1}^{r} \max _{x \in[0,1]^{n}}\left|\left(\sigma \psi_{\nu}^{\prime}\right)(x+m-l) \| \sigma(l) c_{\nu \nu^{\prime}}(l)\right| \\
& \leq \sum_{\nu^{\prime}=1}^{r}\left[\left\|\sigma \psi_{\nu}^{\prime}\right\|_{w}\left(\sum_{l \in \mathbb{Z}^{n}} \sigma(l)\left|c_{\nu \nu^{\prime}}(l)\right|\right)\right]<\infty .
\end{aligned}
$$

Finally, we want to show that the function $\tilde{\psi}_{\nu}$ has the same number of vanishing moments as $\psi_{\nu}$ for all $\nu \in I$.

Definition 2.32. Let $k, m \in \mathbb{N}$ and $\sigma(x)=(1+\|x\|)^{k}, k>m+1$, and let $D^{m}$ be the space defined by $D^{m}:=\left\{f(x): \sigma(x)|f(x)|\right.$ is bounded on $\left.\mathbb{R}^{n}\right\}$. A function $f$ in $D^{m}$ has $N$ vanishing moments with $N \leq m$ if $f$ satisfies

$$
\int_{\mathbb{R}^{n}} x^{p} f(x) d x=0, \quad \text { for all } 0 \leq p \leq N \text { and } \int_{\mathbb{R}^{n}} x^{N+1} f(x) d x \neq 0,
$$

where $p$ is a positive integer.

Theorem 2.33. Let $\psi_{\nu} \in D^{N}$ for $\nu \in I \backslash\{0\}$, and let $\psi_{\nu}$ and $\tilde{\psi}_{\nu}$ have $N_{1}$ and $N_{2}$ vanishing moments, respectively, where $N_{1}, N_{2} \leq N$. Then $N_{1}=N_{2}$.

Proof. Since $\tilde{\psi}_{\nu}$ has $N_{2}$ vanishing moments, we have $\tilde{\psi}_{\nu}(x)=\sum_{\nu^{\prime}=1}^{r} \sum_{l \in \mathbb{Z}^{n}}$ $c_{\nu \nu^{\prime}}(l) \psi_{\nu^{\prime}}(x-l)$ where $\left\{c_{\nu \nu^{\prime}}(l)\right\}_{l \in \mathbb{Z}^{n}}$ is absolutely convergent and

$$
\begin{aligned}
\int_{\mathbb{R}^{n}} x^{p} \tilde{\phi}_{\nu}(x) d x & =\int_{\mathbb{R}^{n}} x^{p}\left(\sum_{\nu^{\prime}=1} \sum_{l \in \mathbb{Z}^{n}} c_{\nu \nu^{\prime}}(l) \psi_{\nu^{\prime}}(x-l)\right) d x \\
& =\sum_{\nu^{\prime}=1} \sum_{l \in \mathbb{Z}^{n}} c_{\nu \nu^{\prime}}(l) \int_{\mathbb{R}^{n}} x^{p} \psi_{\nu^{\prime}}(x-l) d x=0
\end{aligned}
$$

for each $0 \leq p \leq N_{1}$. Thus, $N_{1} \leq N_{2}$. On the other hand, it is easy to prove $\tilde{\psi}_{\nu} \in D^{N}$ from $\psi_{\nu} \in D^{N}$. By Equation (2.23), there exists a sequence $\left\{d_{\nu \nu^{\prime}}(k)\right\} \in l^{1}\left(\mathbb{Z}^{n}\right)$ such that $\psi_{\nu}(x)=\sum_{\nu^{\prime}=1}^{r} \sum_{k \in \mathbb{Z}^{n}} d_{\nu \nu^{\prime}}(k) \tilde{\psi}_{\nu^{\prime}}(x+k)$ and hence we obtain $N_{1} \geq N_{2}$. Thus $N_{1}=N_{2}$.

Example 2.34. Let

$$
X=\left[\begin{array}{lllll}
1 & 1 & 0 & 0 & 1 \\
0 & 0 & 1 & 1 & 1
\end{array}\right]
$$


Then the box spline $B_{X}$ satisfies the 2 -scale dilation equation (2.1) with mask $a$ given by

$$
\tilde{a}(\xi)=2^{-3}\left(1+z_{1}\right)^{2}\left(1+z_{2}\right)^{2}\left(1+z_{1} z_{2}\right) .
$$

$B_{X}$ is $C^{1}$ with compact support and has stable integer translates since the matrix $X$ is unimodular (see [3]). Moreover, $B_{X}$ belongs to $\mathcal{M}\left(\mathbb{R}^{2}\right)$ (see [2]). By (2.5), we have (see [9])

$$
\begin{aligned}
& \widetilde{a^{00}}(z)=\frac{1}{8}\left(1+z_{1}+z_{2}+5 z_{1} z_{2}\right), \\
& \widetilde{a^{10}}(z)=\frac{1}{8}\left(2+4 z_{2}+2 z_{1} z_{2}\right), \\
& \widetilde{a^{01}}(z)=\frac{1}{8}\left(2+4 z_{1}+2 z_{1} z_{2}\right), \\
& \widetilde{a^{10}}(z)=\frac{1}{8}\left(5+z_{1}+z_{2}+z_{1} z_{2}\right) .
\end{aligned}
$$

Furthermore, by using Lemmas $2.11-12$ we see that the function $F(\xi):=$ $\left(\widetilde{a^{\nu}}(\xi): \nu \in I\right)$ is Hölder continuous from $[0,1]^{2}$ into $\mathbb{C}^{2} \backslash\{0\}$ and hence $F$ is extensible; that is, the matrix

$$
\left[\begin{array}{cccc}
\widetilde{a^{00}} & \widetilde{a^{10}} & \widetilde{a^{01}} & \widetilde{a^{11}} \\
1 & 0 & 0 & 0 \\
1 & 4 & 0 & 1 \\
1 & 0 & 4 & 1
\end{array}\right]
$$

has determinant -8 . Using this matrix we can define functions

$$
\begin{aligned}
& \psi_{00}(x):=B_{X}(x), \\
& \psi_{10}(x):=B_{X}(2 x), \\
& \psi_{01}(x):=B_{X}(2 x)+4 B_{X}(2 x+(1,0))+B_{X}(2 x+(1,1)), \\
& \psi_{11}(x):=B_{X}(2 x)+4 B_{X}(2 x+(0,1))+B_{X}(2 x+(1,1))
\end{aligned}
$$

for all $x \in \mathbb{R}^{2}$ and $\psi_{\nu}, \nu \in I$. These have stable integer translates in $\mathcal{V}_{1}$ and are of class $C^{1}\left(\mathbb{R}^{2}\right)$ with compact support. By Theorems 2.12 and 2.20, the decay rate of the bi-orthogonal Riesz bases is the same with that of $\psi_{\nu}$. But, in practice, we have difficulty in computing the Gram-Schmidt orthogonalization process, for instance, since the overlap function of $B_{X}$ is

$$
\left\langle B_{X}, B_{X}\right\rangle(\xi)=\sum_{\beta \in \mathbb{Z}^{2}}\left(\frac{\sin \left(\pi \xi_{1}\right)}{\pi\left(\xi_{1}+\beta_{1}\right)}\right)^{4}\left(\frac{\sin \left(\pi \xi_{2}\right)}{\pi\left(\xi_{2}+\beta_{2}\right)}\right)^{4}\left(\frac{\sin \left(\pi \xi_{1}+\pi \xi_{2}\right)}{\pi\left(\xi_{1}+\beta_{1}+\xi_{2}+\beta_{2}\right)}\right)^{2}
$$


for all $\xi:=\left(\xi_{1}, \xi_{2}\right) \in \mathbb{R}^{2}$. This is hard to put into explicit form as a trigonometric series. The overlap function of a box spline can be expressed by applying Poisson summation formula. Indeed, given the symmetric box spline

$$
M_{X \cup X}:=B_{X} * B_{X}^{*},
$$

where $B_{X}^{*}(x):=B_{X}(-x)$, we have the generalized Euler-Frobenius Laurent polynomial

$$
\left\langle B_{X}, B_{X}\right\rangle(\xi)=\sum_{\beta \in \mathbb{Z}^{2}} M_{X \cup X}(\beta) e^{2 \pi i \beta \cdot \xi} .
$$

Note that (2.36) is finite since the support of $M_{X \cup X}$ is the set

$$
\left\{\sum_{j=1}^{5} x^{j} t_{j}:-1 \leq t_{j} \leq 1 ; j=1,2,3,4,5\right\} .
$$

This implies that the overlap function of $B_{X}$ is a trigonometric polynomial. We may use numerical methods to treat this problem. There is a positive number $\rho=1$ such that $\left\|\sigma B_{X}\right\|_{w}+\left\|\sigma \hat{B}_{X}\right\|_{w}<\infty$ where $\sigma(x)=1+|x|$ and hence for each $\nu \in I \backslash\{0\}, \tilde{\psi}_{\nu} \in C^{1}\left(\mathbb{R}^{2}\right)$ by Corollary 2.27 .

\section{REFERENCES}

1. G. Battle, Cardinal spline interpolation and the block spin construction of wavelets, in Wavelets: A Tutorial in Theory and Applications, C. Chui (ed.), Academic Press, New York, 1992, 73-90.

2. K. F. Chang, Wavelet Analysis on Hilbert Spaces, Ph.D. Dissertation, The University of Texas at Austin, 1993.

3. W. Dahmen and C. A. Micchelli, Translates of multivariate splines, Linear Algebra Appl. 52/53 (1983), 217-234.

4. I. Gelfand, D. Rikov and G. Shilov, Commutative Normed Rings, Chelsea, New York, 1964.

5. T.N.T. Goodman, S. L. Lee, and W. S. Tang, Wavelets in wandering subspaces, Trans. Amer. Math. Soc. 338 (1993), 639-654.

6. V. Guillemin and A. Pollack, Differential Topology, Prentice-Hall, Englewood Cliffs, New Jersey, 1974.

7. E. Hewitt and K. A. Ross, Abstract Harmonic Analysis, Vol. II, SpringerVerlag, Berlin, 1963.

8. J. G. Hocking, Topology, Addison-Wesley, Massachusetts, 1961. 
9. R. Q. Jia and C. A. Micchelli, Using the refinement equations for the construction of pre-wavelets II: Powers of two, in Curves and Surfaces, P. J. Laurent, A. LeMehaute, and L. L. Schumaker (eds.), Academic Press, New York, 1991, 209-246.

10. R. Q. Jia and C. A. Micchelli, Using the refinement equations for the construction of pre-wavelet V, Computing 48 (1992), 61-72.

11. J. Lei, $L_{p}$-approximation by certain projection operators, J. Math. Anal. Appl. 185 (1994), 1-14.

12. B. S. Mitjagin, Maximal ideals of certain normed rings, Sibirsk. Mat. Z. 1 (1960), (Russian).

13. W. Rudin, Functional Analysis, McGraw-Hill, New York, 1973.

Department of Applied Mathematics, Feng Chia University, Tai-Chung, Taiwan 